\title{
Fractional operators with generalized Mittag-Leffler $k$-function
}

\author{
Shahid Mubeen ${ }^{1 *}$ (1) and Rana Safdar Ali $^{1}$
}

\section{"Correspondence:} smjhanda@gmail.com

'Department of Mathematics, University of Sargodha, Sargodha, Pakistan

\section{Springer}

\begin{abstract}
In this paper, our main aim is to deal with two integral transforms involving the Gauss hypergeometric functions as their kernels. We prove some composition formulas for such generalized fractional integrals with Mittag-Leffler $k$-function. The results are established in terms of the generalized Wright hypergeometric function. The Euler integral $k$-transformation for Mittag-Leffler $k$-functions has also been developed.
\end{abstract}

Keywords: Fractional integral operators; Generalized Mittag-Leffler k-function; Generalized Wright function

\section{Introduction}

Mittag-Leffler functions are important in studying solutions of fractional differential equations, and they are associated with a wide range of problems in many areas of mathematics and physics. The importance and great considerations of Mittag-Leffler functions have led many researchers in the theory of special functions to exploring possible generalizations and applications. Many more extensions or unifications for these functions are found in a large number of papers [1-5]. A useful generalization of the Mittag-Leffler function, the so-called Mittag-Leffler $k$-function has been introduced and studied in [6]. Many mathematicians discussed and obtained new results [7-13], seen as theoretical developments to the fractional operators. These considerations have led various researchers in the field of special functions for exploring possible extensions of and applications to the MittagLeffler function. Recently, fractional calculus gained more attention due to its wide variety of applications in various fields [14-18]. In the literature of fractional calculus, it is distinctly observed that the fractional integral operators and fractional integral formulas containing special functions occupied an influential place in computational and applied mathematics [19-21]. The fractional calculus of various types of special functions is used in many research papers [22-25]. For more details about the recent works in the field of dynamic system theory, stochastic systems, nonequilibrium statistical mechanics, and quantum mechanics, we refer the interesting readers to [26-32]. Throughout this paper, we denote by $\mathbb{C}, \mathbb{N}, \mathbb{R}^{+}$, and $\mathbb{R}$ the sets of complex numbers, natural numbers, positive real numbers, and real numbers, respectively.

(c) The Author(s) 2019. This article is licensed under a Creative Commons Attribution 4.0 International License, which permits use sharing, adaptation, distribution and reproduction in any medium or format, as long as you give appropriate credit to the original author(s) and the source, provide a link to the Creative Commons licence, and indicate if changes were made. The images or other third party material in this article are included in the article's Creative Commons licence, unless indicated otherwise in a credit line to the material. If material is not included in the article's Creative Commons licence and your intended use is not permitted by statutory regulation or exceeds the permitted use, you will need to obtain permission directly from the copyright holder. To view a copy of this licence, visit http://creativecommons.org/licenses/by/4.0/. 
The Gauss hypergeometric function is defined as follows [33]: for all $d, e, f \in \mathbb{C}, f \neq$ $0,-1,-2, \ldots$, and $|z|<1$,

$$
{ }_{2} F_{1}(d, e ; f ; z)=\sum_{n=0}^{\infty} \frac{(d)_{n}(e)_{n}}{(f)_{n}} \frac{z^{n}}{n !}
$$

where $(d)_{n},(e)_{n}$, and $(f)_{n}$ are the Pochhammer symbols.

The Pochhammer symbols are defined as [34]

$$
(l)_{n}= \begin{cases}1 & \text { for } n=0, l \neq 0, \\ l(l+1)(l+2) \cdots(l+n-1) & \text { for } n \geq 1,\end{cases}
$$

where $l \in \mathbb{C}$ and $n \in \mathbb{N}$.

The gamma function [34] for $\Re(u)>0$ is defined as

$$
\Gamma(z)=\int_{0}^{\infty} t^{z-1} e^{-t} d t
$$

The beta function [34] is defined as

$$
\beta(l, h)=\int_{0}^{1} t^{l-1}(1-t)^{h-1} d t, \quad \Re(l)>0, \Re(h)>0 .
$$

The beta $k$-function [33] is defined as

$$
\beta_{k}(l, h)=\frac{1}{k} \int_{0}^{1} s^{\frac{l}{k}-1}(1-s)^{\frac{h}{k}-1} d s, \quad \Re(l)>0, \Re(h)>0 .
$$

The generalized fractional integration operators are defined for $u>0, d, e, f \in \mathbb{C}$, and $\Re(d)>0$ as follows (see [35-37]):

$$
\left(I_{0, u}^{a, b, c} h\right)(u)=\frac{u^{-a-b}}{\Gamma(a)} \int_{0}^{u}(u-t)^{a-1}{ }_{2} F_{1}\left(a+b,-c ; a ; 1-\frac{t}{u}\right) h(t) d t
$$

and

$$
\left(I_{z, \infty}^{d, e, f} h\right)(z)=\frac{1}{\Gamma(d)} \int_{z}^{\infty}(t-z)^{d-1} t^{-d-e}{ }_{2} F_{1}\left(d+e,-f ; d ; 1-\frac{z}{t}\right) h(t) d t,
$$

where $\Gamma$ is the gamma function [38], and ${ }_{2} F_{1}$ is the hypergeometric series defined by Rainville [39].

The Mittag-Leffler function $E_{\alpha}(z)$ is defined by [40, 41]

$$
E_{\alpha}(z)=\sum_{n=0}^{\infty} \frac{z^{n}}{\Gamma(\alpha n+1)}
$$

for $z \in \mathbb{C}$ and $\alpha \geq 0$. The Mittag-Leffler function $E_{\alpha}(z)$ has been extended in a number of ways and, together with its extensions, applied in various research areas such as engineering and (in particular) statistics. The Mittag-Leffler functions and related distributions were given in [32]. 
The generalization of $E_{\alpha}(z)$, also known as the Wiman function [42], is given by

$$
E_{\alpha, \beta}(z)=\sum_{n=0}^{\infty} \frac{z^{n}}{\Gamma(\alpha n+\beta)}
$$

for $\alpha, \beta \in \mathbb{C}$ with $\Re(\alpha)>0, \Re(\beta)>0$.

In 1971, Prabhakar [43] proposed the more general function

$$
E_{v, \rho}^{\delta}(z)=\sum_{n=0}^{\infty} \frac{(\delta)_{n} z^{n}}{\Gamma(v n+\rho) n !} .
$$

A useful generalization of the Mittag-Leffler, the so-called Mittag-Leffler $k$-function has been introduced and studied in [2, 6]. The generalized Mittag-Leffler $k$-function [44] is defined as

$$
E_{k, v, \rho}^{\delta}(t)=\sum_{n=0}^{\infty} \frac{(\delta)_{n, k} t^{n}}{\Gamma_{k}(v n+\rho) n !},
$$

for $k \in \mathbb{R}^{+}, v, \rho, \delta, t \in \mathbb{C}$ with $\Re(v)>0, \Re(\rho)>0$.

The integral form of the generalized gamma $k$-function is given by [45]

$$
\Gamma_{k}(z)=\int_{0}^{\infty} t^{z-1} e^{\frac{-t^{k}}{k}} d t
$$

for $k \in \mathbb{R}^{+}$and $z \in \mathbb{C}$ with $\operatorname{Re}(z)>0$. By inspection we conclude the following relations:

$$
\begin{aligned}
& \Gamma_{k}(z+k)=k \Gamma_{k}(z), \\
& \Gamma_{k}(\gamma)=(k)^{\frac{\gamma}{k}-1} \Gamma\left(\frac{\gamma}{k}\right) .
\end{aligned}
$$

If $k$ approaches one, then the generalized Mittag-Leffler $k$-function reduces to the generalized Mittag-Leffler function.

The generalized hypergeometric function is defined as [46]

$$
{ }_{p} F_{q}\left(d_{1}, \ldots, d_{p}, e_{1}, \ldots, e_{q} ; t\right)=\sum_{n=0}^{\infty} \frac{\left(d_{1}\right)_{n} \cdots\left(d_{p}\right)_{n}}{\left(e_{1}\right)_{n} \cdots\left(e_{q}\right)_{n}} \frac{t^{n}}{n !}
$$

where $d_{i}, e_{j} \in \mathbb{C}, e_{j} \neq 0,-1, \ldots(i=1,2, \ldots, p ; j=1,2, \ldots, q)$.

The generalized Wright hypergeometric function is defined as [47]

$$
{ }_{l} \psi_{h}(t)={ }_{l} \Psi_{h}\left[\begin{array}{c}
\left(c_{i}, p_{i}\right)_{1, l} \\
\left(d_{j}, q_{j}\right){ }_{1, h}
\end{array} \mid t\right] \equiv \sum_{n=0}^{\infty} \frac{\prod_{i=1}^{l} \Gamma\left(c_{i}+p_{i} n\right) t^{n}}{\prod_{j=1}^{h} \Gamma\left(d_{j}+q_{j} n\right) n !}
$$

where $t \in \mathbb{C}, c_{i}, d_{j} \in \mathbb{C}$, and $p_{i}, q_{j} \in \mathbb{R}(i=1,2, \ldots, l ; j=1,2, \ldots, h)$.

The following identity of Gauss hypergeometric function holds:

$$
{ }_{2} F_{1}(e, f ; d ; 1)=\frac{\Gamma(d) \Gamma(d-e-f)}{\Gamma(d-e) \Gamma(d-f)}, \quad \Re(d-e-f)>0 .
$$


The hypergeometric $k$-function [48] is defined as

$$
{ }_{2} F_{1, k}\left(\left(\alpha^{\prime}, k\right),\left(\beta^{\prime}, k\right) ;\left(\eta^{\prime}, k\right) ; t\right)=\sum_{m=0}^{\infty} \frac{\left(\alpha^{\prime}\right)_{m, k}\left(\beta^{\prime}\right)_{m, k}}{\left(\eta^{\prime}\right)_{m, k}} \frac{t^{m}}{m !}, \quad k>0,
$$

where

$$
\alpha^{\prime}, \beta^{\prime}, \eta^{\prime} \in \mathbb{C}, \quad \eta^{\prime} \neq 0,-1,-2,-3, \ldots, \quad|t|<1 .
$$

\section{Preliminary lemmas}

In this section, we derive the fundamental results of left- and right-sided generalized $k$ fractional integration with power $k$-function. The following lemmas proved in [35] are needed to prove our main results.

Lemma 1 ([35]) For $a, b, c, \rho \in \mathbb{C}$ with

$$
\begin{aligned}
& \Re(a)>0 \quad \text { and } \quad \Re(\rho+c-b)>0 \\
& \left(I_{0, u}^{a, b, c} t^{\rho-1}\right)(u)=u^{\rho-b-1} \frac{\Gamma(\rho) \Gamma(\rho+c-b)}{\Gamma(\rho-b) \Gamma(\rho+a+c)} .
\end{aligned}
$$

Lemma 2 ([35]) For $a, b, c \in \mathbb{C}$ with

$$
\Re(a)>0 \quad \text { and } \quad \mathfrak{R}(\rho)<1+\min [\Re(b), \mathfrak{R}(c)]
$$

we have

$$
\left(I_{u, \infty}^{a, b, c} t^{\rho-1}\right)(u)=u^{\rho-b-1} \frac{\Gamma(b-\rho+1) \Gamma(c-\rho+1)}{\Gamma(1-\rho) \Gamma(a+b+c-\rho+1)} .
$$

Theorem 1 Let $\alpha^{\prime}, \beta^{\prime}, \eta^{\prime} \in \mathbb{C}, k \in \mathbb{R}^{+}$with $\mathfrak{R}\left(\alpha^{\prime}\right)>0$ and $\mathfrak{R}\left(\sigma^{\prime}\right)>\max \left[0, \Re\left(\beta^{\prime}-\eta^{\prime}\right)\right]$. Then

$$
\left(I_{0, y}^{\alpha^{\prime}, \beta^{\prime}, \eta^{\prime}} S^{\frac{\sigma^{\prime}}{k}-1}\right)_{k}(y)=y^{\frac{\sigma^{\prime}-\beta^{\prime}}{k}-1} \frac{\Gamma_{k}\left(\sigma^{\prime}\right) \Gamma_{k}\left(\sigma^{\prime}+\eta^{\prime}-\beta^{\prime}\right)}{\Gamma_{k}\left(\sigma^{\prime}-\beta^{\prime}\right) \Gamma_{k}\left(\sigma^{\prime}+\alpha^{\prime}+\eta^{\prime}\right)} .
$$

Proof Consider the left-sided generalized $k$-fractional integral operator

$$
\begin{aligned}
\left(I_{0, y}^{\alpha^{\prime}, \beta^{\prime}, \eta^{\prime}} g\right)_{k}(y)= & \frac{y^{\frac{-\alpha^{\prime}-\beta^{\prime}}{k}}}{k \Gamma_{k}\left(\alpha^{\prime}\right)} \int_{0}^{y}(y-s)^{\frac{\alpha^{\prime}}{k}-1} \\
& \times{ }_{2} F_{1, k}\left(\left(\alpha^{\prime}+\beta^{\prime}, k\right),\left(-\eta^{\prime}, k\right) ;\left(\alpha^{\prime}, k\right) ; 1-\frac{s}{y}\right) g(s) d s .
\end{aligned}
$$

Using the power $k$-function in equation (19), we have

$$
\begin{aligned}
\left(I_{0, y}^{\alpha^{\prime}, \beta^{\prime}, \eta^{\prime}} s^{\frac{\sigma^{\prime}}{k}-1}\right)_{k}(y)= & \frac{y^{\frac{-\alpha^{\prime}-\beta^{\prime}}{k}}}{k \Gamma_{k}\left(\alpha^{\prime}\right)} \int_{0}^{y}(y-s)^{\frac{\alpha^{\prime}}{k}-1} \\
& \times{ }_{2} F_{1, k}\left(\left(\alpha^{\prime}+\beta^{\prime}, k\right),\left(-\eta^{\prime}, k\right) ;\left(\alpha^{\prime}, k\right) ; 1-\frac{s}{y}\right) s^{\frac{\sigma^{\prime}}{k}-1} d s .
\end{aligned}
$$


Using equation (18) in equation (20), we get

$$
\begin{aligned}
\left(I_{0, y}^{\alpha^{\prime}, \beta^{\prime}, \eta^{\prime}} s^{\frac{\sigma^{\prime}}{k}-1}\right)_{k}(y)= & \frac{y^{\frac{-\alpha^{\prime}-\beta^{\prime}}{k}}}{k \Gamma_{k}\left(\alpha^{\prime}\right)} \int_{0}^{y}(y-s)^{\frac{\alpha^{\prime}}{k}-1} \\
& \times \sum_{m=0}^{\infty} \frac{\left(\alpha^{\prime}+\beta^{\prime}\right)_{m, k}\left(-\eta^{\prime}\right)_{m, k}}{\left(\alpha^{\prime}\right)_{m, k} m !}\left(1-\frac{s}{y}\right)^{m} s^{\frac{\sigma^{\prime}}{k}-1} d s .
\end{aligned}
$$

By putting

$$
\begin{aligned}
& s=v y \quad \Longrightarrow \quad d s=y d v, \\
& s=0 \quad \Longrightarrow \quad v=0, \\
& s=y \quad \Longrightarrow \quad v=1
\end{aligned}
$$

in equation (21), we obtain

$$
\begin{aligned}
& \left(I_{0, y}^{\alpha^{\prime}, \beta^{\prime}, \eta^{\prime}} s^{\frac{\sigma^{\prime}}{k}-1}\right)_{k}(y) \\
& =\frac{y^{\frac{-\alpha^{\prime}-\beta^{\prime}}{k}}}{k \Gamma\left(\alpha^{\prime}\right)} \int_{0}^{1}(y-v y)^{\frac{\alpha^{\prime}}{k}-1}(1-v)^{m}(v y)^{\frac{\sigma^{\prime}}{k}-1} \sum_{m=0}^{\infty} \frac{\left(\alpha^{\prime}+\beta^{\prime}\right)_{m, k}\left(-\eta^{\prime}\right)_{m, k}}{\left(\alpha^{\prime}\right)_{m, k} m !} y d v \\
& =\frac{y^{\frac{-\alpha^{\prime}-\beta^{\prime}}{k}}}{k \Gamma\left(\alpha^{\prime}\right)} \sum_{m=0}^{\infty} \frac{\left(\alpha^{\prime}+\beta^{\prime}\right)_{m, k}\left(-\eta^{\prime}\right)_{m, k}}{\left(\alpha^{\prime}\right)_{m, k} m !} \int_{0}^{1} y^{\frac{\alpha^{\prime}}{k}-1}(1-v)^{\frac{\alpha^{\prime}}{k}-1}(1-v)^{m} v^{\frac{\sigma^{\prime}}{k}-1} y^{\frac{\sigma^{\prime}}{k}-1} y d v \\
& =\frac{y^{\frac{-\alpha^{\prime}-\beta^{\prime}}{k}}}{k \Gamma\left(\alpha^{\prime}\right)} \sum_{m=0}^{\infty} \frac{\left(\alpha^{\prime}+\beta^{\prime}\right)_{m, k}\left(-\eta^{\prime}\right)_{m, k}}{\left(\alpha^{\prime}\right)_{m, k} m !} \int_{0}^{1} y^{\frac{\alpha^{\prime}}{k}-1+\frac{\sigma^{\prime}}{k}-1+1}(1-v)^{\frac{\alpha^{\prime}}{k}+m-1} v^{\frac{\sigma^{\prime}}{k}-1} d v \\
& =\frac{y^{-\frac{\alpha^{\prime}}{k}-\frac{\beta^{\prime}}{k}+\frac{\alpha^{\prime}}{k}+\frac{\sigma^{\prime}}{k}-1}}{k \Gamma\left(\alpha^{\prime}\right)} \sum_{m=0}^{\infty} \frac{\left(\alpha^{\prime}+\beta^{\prime}\right)_{m, k}\left(-\eta^{\prime}\right)_{m, k}}{\left(\alpha^{\prime}\right)_{m, k} m !} \int_{0}^{1}(1-v)^{\frac{\alpha^{\prime}}{k}+m-1} v^{\frac{\sigma^{\prime}}{k}-1} d v \\
& =\frac{y^{\frac{\sigma^{\prime}-\beta^{\prime}}{k}-1}}{\Gamma_{k}\left(\alpha^{\prime}\right)} \sum_{m=0}^{\infty} \frac{\left(\alpha^{\prime}+\beta^{\prime}\right)_{m, k}\left(-\eta^{\prime}\right)_{m, k}}{\left(\alpha^{\prime}\right)_{m, k} m !} \frac{1}{k} \int_{0}^{1}(1-v)^{\frac{\alpha^{\prime}+m k}{k}-1} v^{\frac{\sigma^{\prime}}{k}-1} d v .
\end{aligned}
$$

Since

$$
\beta_{k}(l, h)=\frac{\Gamma_{k}(l) \Gamma_{k}(h)}{\Gamma_{k}(l+h)},
$$

by equations (5) and (22) we have

$$
=\frac{y^{\frac{\sigma^{\prime}-\beta^{\prime}}{k}-1}}{\Gamma_{k}\left(\alpha^{\prime}\right)} \sum_{m=0}^{\infty} \frac{\left(\alpha^{\prime}+\beta^{\prime}\right)_{m, k}\left(-\eta^{\prime}\right)_{m, k}}{\left(\alpha^{\prime}\right)_{m, k} m !} \frac{\Gamma_{k}\left(\alpha^{\prime}+m k\right) \Gamma_{k}\left(\sigma^{\prime}\right)}{\Gamma_{k}\left(\alpha^{\prime}+\sigma^{\prime}+m k\right)} .
$$

Since

$$
\Gamma_{k}(t+m k)=(t)_{m, k} \Gamma_{k}(t)
$$


from equation (24) we get

$$
\begin{aligned}
\left(I_{0, y}^{\alpha^{\prime}, \beta^{\prime}, \eta^{\prime}} s^{\frac{\sigma^{\prime}}{k}-1}\right)_{k}(y) & =\frac{y^{\frac{\sigma^{\prime}-\beta^{\prime}}{k}-1}}{\Gamma_{k}\left(\alpha^{\prime}\right)} \sum_{m=0}^{\infty} \frac{\left(\alpha^{\prime}+\beta^{\prime}\right)_{m, k}\left(-\eta^{\prime}\right)_{m, k}}{\left(\alpha^{\prime}\right)_{m, k} m !} \frac{\left(\alpha^{\prime}\right)_{m, k} \Gamma_{k}\left(\alpha^{\prime}\right) \Gamma_{k}\left(\sigma^{\prime}\right)}{\left(\alpha^{\prime}+\sigma^{\prime}\right)_{m, k} \Gamma_{k}\left(\alpha^{\prime}+\sigma^{\prime}\right)} \\
& =y^{\frac{\sigma^{\prime}-\beta^{\prime}}{k}-1} \frac{\Gamma_{k}\left(\sigma^{\prime}\right)}{\Gamma_{k}\left(\alpha^{\prime}+\sigma^{\prime}\right)} \sum_{m=0}^{\infty} \frac{\left(\alpha^{\prime}+\beta^{\prime}\right)_{m, k}\left(-\eta^{\prime}\right)_{m, k}}{\left(\alpha^{\prime}+\sigma^{\prime}\right)_{m, k} m !}
\end{aligned}
$$

Using equation (18), from equation (26) we have

$$
\left(I_{0, y}^{\alpha^{\prime}, \beta^{\prime}, \eta^{\prime}} s^{\sigma^{\prime}-1}\right)_{k}(y)=y^{\frac{\sigma^{\prime}-\beta^{\prime}}{k}-1} \frac{\Gamma_{k}\left(\sigma^{\prime}\right)}{\Gamma_{k}\left(\alpha^{\prime}+\sigma^{\prime}\right)}{ }_{2} F_{1, k}\left(\left(\alpha^{\prime}+\beta^{\prime}, k\right),\left(-\eta^{\prime}, k\right) ;\left(\alpha^{\prime}+\sigma^{\prime}, k\right) ; 1\right) .
$$

We can also write

$$
\begin{aligned}
\left(I_{0, y}^{\alpha^{\prime}, \beta^{\prime}, \eta^{\prime}} s^{\frac{\sigma^{\prime}}{k}-1}\right)_{k}(y)= & y^{\frac{\sigma^{\prime}-\beta^{\prime}}{k}-1} \frac{\Gamma_{k}\left(\sigma^{\prime}\right)}{\Gamma_{k}\left(\alpha^{\prime}+\sigma^{\prime}\right)} \\
& \times{ }_{2} F_{1, k}\left(\left(\alpha^{\prime}+\beta^{\prime}, k\right),\left(-\eta^{\prime}, k\right) ;\left(\alpha^{\prime}+\sigma^{\prime}, k\right) ; 1\right) .
\end{aligned}
$$

Since

$$
{ }_{2} F_{1, k}\left[\left(\alpha^{\prime}, k\right),\left(\beta^{\prime}, k\right)\left(\eta^{\prime}, k\right) ; 1\right]=\frac{\Gamma_{k}\left(\eta^{\prime}\right) \Gamma_{k}\left(\eta^{\prime}-\beta^{\prime}-\alpha^{\prime}\right)}{\Gamma_{k}\left(\eta^{\prime}-\alpha^{\prime}\right) \Gamma_{k}\left(\eta^{\prime}-\beta^{\prime}\right)},
$$

from equation (27) we obtain

$$
\begin{aligned}
& \left(I_{0, y}^{\alpha^{\prime}, \beta^{\prime}, \eta^{\prime}} S^{\frac{\sigma^{\prime}}{k}-1}\right)_{k}(y)=y^{\frac{\sigma^{\prime}-\beta^{\prime}}{k}-1} \frac{\Gamma_{k}\left(\sigma^{\prime}\right)}{\Gamma_{k}\left(\alpha^{\prime}+\sigma^{\prime}\right)} \frac{\Gamma_{k}\left(\alpha^{\prime}+\sigma^{\prime}\right) \Gamma_{k}\left(\alpha^{\prime}+\sigma^{\prime}-\alpha^{\prime}-\beta^{\prime}+\eta^{\prime}\right)}{\Gamma_{k}\left(\alpha^{\prime}+\sigma^{\prime}-\alpha^{\prime}-\beta^{\prime}\right) \Gamma_{k}\left(\alpha^{\prime}+\sigma^{\prime}+\eta^{\prime}\right)}, \\
& \left(I_{0, y}^{\alpha^{\prime}, \beta^{\prime}, \eta^{\prime}} s^{\frac{\sigma^{\prime}}{k}-1}\right)_{k}(y)=y^{\frac{\sigma^{\prime}-\beta^{\prime}}{k}-1} \frac{\Gamma_{k}\left(\sigma^{\prime}\right) \Gamma_{k}\left(\sigma^{\prime}+\eta^{\prime}-\beta^{\prime}\right)}{\Gamma_{k}\left(\sigma^{\prime}-\beta^{\prime}\right) \Gamma_{k}\left(\sigma^{\prime}+\alpha^{\prime}+\eta^{\prime}\right)} .
\end{aligned}
$$

Theorem 2 Let $\alpha^{\prime}, \beta^{\prime}, \eta^{\prime} \in \mathbb{C}$. Then

$$
\left(I_{y, \infty}^{\alpha^{\prime}, \beta^{\prime}, \eta^{\prime}} s^{\frac{\sigma^{\prime}}{k}-1}\right)_{k}(y)=y^{\frac{\sigma^{\prime}-\beta^{\prime}}{k}-1} \frac{\Gamma_{k}\left(\beta^{\prime}-\sigma^{\prime}+k\right) \Gamma_{k}\left(-\sigma^{\prime}+k+\eta^{\prime}\right)}{\Gamma_{k}\left(-\sigma^{\prime}+k\right) \Gamma_{k}\left(\alpha^{\prime}-\sigma^{\prime}+\beta^{\prime}+k+\eta^{\prime}\right)} .
$$

Proof Consider the right-sided generalized $k$-fractional integral operator

$$
\begin{aligned}
\left(I_{y, \infty}^{\alpha^{\prime}, \beta^{\prime}, \eta^{\prime}} g\right)_{k}(y)= & \frac{1}{k \Gamma_{k}\left(\alpha^{\prime}\right)} \int_{y}^{\infty}(s-y)^{\frac{\alpha^{\prime}}{k}-1} s^{\frac{-\alpha^{\prime}-\beta^{\prime}}{k}} \\
& \times{ }_{2} F_{1, k}\left(\left(\alpha^{\prime}+\beta^{\prime}, k\right),\left(-\eta^{\prime}, k\right) ;\left(\alpha^{\prime}, k\right) ; 1-\frac{y}{s}\right) g(s) d s .
\end{aligned}
$$

Using the power $k$-function in (30), we have

$$
\begin{aligned}
\left(I_{y, \infty}^{\alpha^{\prime}, \beta^{\prime}, \eta^{\prime}} s^{\frac{\sigma^{\prime}}{k}-1}\right)_{k}(y)= & \frac{1}{k \Gamma_{k}\left(\alpha^{\prime}\right)} \int_{y}^{\infty}(s-y)^{\frac{\alpha^{\prime}}{k}-1} s^{\frac{-\alpha^{\prime}-\beta^{\prime}}{k}} \\
& \times{ }_{2} F_{1, k}\left(\left(\alpha^{\prime}+\beta^{\prime}, k\right),\left(-\eta^{\prime}, k\right) ;\left(\alpha^{\prime}, k\right) ; 1-\frac{y}{s}\right) s^{\frac{\sigma^{\prime}}{k}-1} d s .
\end{aligned}
$$


Using equation (18) in equation (31), we get

$$
\begin{aligned}
\left(I_{y, \infty}^{\alpha^{\prime}, \beta^{\prime}, \eta^{\prime}} s^{\frac{\sigma^{\prime}}{k}-1}\right)_{k}(y)= & \frac{1}{k \Gamma_{k}\left(\alpha^{\prime}\right)} \int_{y}^{\infty}(s-y)^{\frac{\alpha^{\prime}}{k}-1} s^{\frac{-\alpha^{\prime}-\beta^{\prime}}{k}} \\
& \times \sum_{m=0}^{\infty} \frac{\left(\alpha^{\prime}+\beta^{\prime}\right)_{m, k}\left(-\eta^{\prime}\right)_{m, k}}{\left(\alpha^{\prime}\right)_{m, k} m !}\left(1-\frac{y}{s}\right)^{m} s^{\frac{\sigma^{\prime}}{k}-1} d s
\end{aligned}
$$

Putting

$$
\begin{aligned}
& s=\frac{y}{v} \quad \Longrightarrow \quad d s=-\frac{y}{v^{2}} d v, \\
& s=y \quad \Longrightarrow \quad v=1, \\
& s=\infty \quad \Longrightarrow \quad v=0
\end{aligned}
$$

in equation (32), we obtain

$$
\begin{aligned}
& \left(I_{y, \infty}^{\alpha^{\prime}, \beta^{\prime}, \eta^{\prime}} s^{\frac{\sigma^{\prime}}{k}-1}\right)_{k}(y) \\
& =\frac{1}{k \Gamma_{k}\left(\alpha^{\prime}\right)} \int_{1}^{0}\left(\frac{y}{v}-y\right)^{\frac{\alpha^{\prime}}{k}-1}\left(\frac{y}{v}\right)^{\frac{-\alpha^{\prime}-\beta^{\prime}}{k}} \sum_{m=0}^{\infty} \frac{\left(\alpha^{\prime}+\beta^{\prime}\right)_{m, k}\left(-\eta^{\prime}\right)_{m, k}}{\left(\alpha^{\prime}\right)_{m, k} m !} \\
& \times(1-v)^{m}\left(\frac{y}{v}\right)^{\frac{\sigma^{\prime}}{k}-1}\left(-\frac{y}{v^{2}}\right) d v \\
& =\frac{1}{k \Gamma_{k}\left(\alpha^{\prime}\right)} \sum_{m=0}^{\infty} \frac{\left(\alpha^{\prime}+\beta^{\prime}\right)_{m, k}\left(-\eta^{\prime}\right)_{m, k}}{\left(\alpha^{\prime}\right)_{m, k} m !} \int_{0}^{1} y^{\frac{\alpha^{\prime}}{k}-1}\left(\frac{1-v}{v}\right)^{\frac{\alpha^{\prime}}{k}-1} y^{\frac{-\alpha^{\prime}-\beta^{\prime}}{k}} v^{\frac{\alpha^{\prime}+\beta^{\prime}}{k}} \\
& \times(1-v)^{m} y^{\frac{\sigma^{\prime}}{k}-1} v^{1-\frac{\sigma^{\prime}}{k}} y v^{-2} d v, \\
& \left(I_{y, \infty}^{\alpha^{\prime}, \beta^{\prime}, \eta^{\prime}} S^{\frac{\sigma^{\prime}}{k}-1}\right)_{k}(y) \\
& =\frac{1}{k \Gamma_{k}\left(\alpha^{\prime}\right)} \sum_{m=0}^{\infty} \frac{\left(\alpha^{\prime}+\beta^{\prime}\right)_{m, k}\left(-\eta^{\prime}\right)_{m, k}}{\left(\alpha^{\prime}\right)_{m, k} m !} \int_{0}^{1} y^{\frac{\alpha^{\prime}}{k}-1-\frac{\alpha^{\prime}}{k}-\frac{\beta^{\prime}}{k}+\frac{\sigma^{\prime}}{k}-1+1}(1-v)^{\frac{\alpha^{\prime}}{k}+m-1} \\
& \times v^{1-\frac{\alpha^{\prime}}{k}+\frac{\alpha^{\prime}}{k}+\frac{\beta^{\prime}}{k}+1-\frac{\sigma^{\prime}}{k}-2} d v \\
& =\frac{y^{\frac{\sigma^{\prime}}{k}-\frac{\beta^{\prime}}{k}-1}}{k \Gamma_{k}\left(\alpha^{\prime}\right)} \sum_{m=0}^{\infty} \frac{\left(\alpha^{\prime}+\beta^{\prime}\right)_{m, k}\left(-\eta^{\prime}\right)_{m, k}}{\left(\alpha^{\prime}\right)_{m, k} m !} \int_{0}^{1}(1-v)^{\frac{\alpha^{\prime}}{k}+m-1} v^{\frac{\beta^{\prime}-\sigma^{\prime}}{k}} d v \\
& =\frac{y^{\frac{\sigma^{\prime}-\beta^{\prime}}{k}-1}}{\Gamma_{k}\left(\alpha^{\prime}\right)} \sum_{m=0}^{\infty} \frac{\left(\alpha^{\prime}+\beta^{\prime}\right)_{m, k}\left(-\eta^{\prime}\right)_{m, k}}{\left(\alpha^{\prime}\right)_{m, k} m !} \frac{1}{k} \int_{0}^{1}(1-v)^{\frac{\alpha^{\prime}+m k}{k}-1} v^{\frac{\beta^{\prime}-\sigma^{\prime}}{k}+1-1} d v .
\end{aligned}
$$

Using equation (5) and equation (23) in equation (33), we get

$$
\begin{aligned}
\left(I_{y, \infty}^{\alpha^{\prime}, \beta^{\prime}, \eta^{\prime}} S^{\frac{\sigma^{\prime}}{k}-1}\right)_{k}(y)= & \frac{y^{\frac{\sigma^{\prime}-\beta^{\prime}}{k}-1}}{\Gamma_{k}\left(\alpha^{\prime}\right)} \\
& \times \sum_{m=0}^{\infty} \frac{\left(\alpha^{\prime}+\beta^{\prime}\right)_{m, k}\left(-\eta^{\prime}\right)_{m, k}}{\left(\alpha^{\prime}\right)_{m, k} m !} \frac{\Gamma_{k}\left(\alpha^{\prime}+m k\right) \Gamma_{k}\left(\beta^{\prime}-\sigma^{\prime}+k\right)}{\Gamma_{k}\left(\alpha^{\prime}+\beta^{\prime}-\sigma^{\prime}+m k+k\right)}
\end{aligned}
$$


Using equation (25) in equation (34), we obtain

$$
\begin{aligned}
& \left(I_{y, \infty}^{\alpha^{\prime}, \beta^{\prime}, \eta^{\prime}} S^{\frac{\sigma^{\prime}}{k}-1}\right)_{k}(y) \\
& =\frac{y^{\frac{\sigma^{\prime}-\beta^{\prime}}{k}}-1}{\Gamma_{k}\left(\alpha^{\prime}\right)} \sum_{m=0}^{\infty} \frac{\left(\alpha^{\prime}+\beta^{\prime}\right)_{m, k}\left(-\eta^{\prime}\right)_{m, k}}{\left(\alpha^{\prime}\right)_{m, k} m !} \frac{\left(\alpha^{\prime}\right)_{m, k} \Gamma_{k}\left(\alpha^{\prime}\right) \Gamma_{k}\left(\beta^{\prime}-\sigma^{\prime}+k\right)}{\left(\alpha^{\prime}-\sigma^{\prime}+\beta^{\prime}+k\right)_{m, k} \Gamma_{k}\left(\alpha^{\prime}-\sigma^{\prime}+\beta^{\prime}+k\right)} \\
& =y^{\frac{\sigma^{\prime}-\beta^{\prime}}{k}-1} \frac{\Gamma_{k}\left(\beta^{\prime}-\sigma^{\prime}+k\right)}{\Gamma_{k}\left(\alpha^{\prime}-\sigma^{\prime}+\beta^{\prime}+k\right)} \sum_{m=0}^{\infty} \frac{\left(\alpha^{\prime}+\beta^{\prime}\right)_{m, k}\left(-\eta^{\prime}\right)_{m, k}}{\left(\alpha^{\prime}-\sigma^{\prime}+\beta^{\prime}+k\right)_{m, k} m !} .
\end{aligned}
$$

Using equation (18) in equation (35), we have

$$
\begin{aligned}
\left(I_{y, \infty}^{\alpha^{\prime}, \beta^{\prime}, \eta^{\prime}} S^{\frac{\sigma^{\prime}}{k}-1}\right)_{k}(y)= & y^{\frac{\sigma^{\prime}-\beta^{\prime}}{k}-1} \frac{\Gamma_{k}\left(\beta^{\prime}-\sigma^{\prime}+k\right)}{\Gamma_{k}\left(\alpha^{\prime}-\sigma^{\prime}+\beta^{\prime}+k\right)} \\
& \times{ }_{2} F_{1, k}\left(\left(\alpha^{\prime}+\beta^{\prime}, k\right),\left(-\eta^{\prime}, k\right) ;\left(\alpha^{\prime}-\sigma^{\prime}+\beta^{\prime}+k, k\right) ; 1\right) .
\end{aligned}
$$

Using equation (28) in equation (36), we get

$$
\begin{aligned}
\left(I_{y, \infty}^{\alpha^{\prime}, \beta^{\prime}, \eta^{\prime}} S^{\frac{\sigma^{\prime}}{k}-1}\right)_{k}(y)= & y^{\frac{\sigma^{\prime}-\beta^{\prime}}{k}-1} \frac{\Gamma_{k}\left(\beta^{\prime}-\sigma^{\prime}+k\right)}{\Gamma_{k}\left(\alpha^{\prime}-\sigma^{\prime}+\beta^{\prime}+k\right)} \\
& \times \frac{\Gamma_{k}\left(\alpha^{\prime}-\sigma^{\prime}+\beta^{\prime}+k\right) \Gamma_{k}\left(\alpha^{\prime}-\sigma^{\prime}+\beta^{\prime}+k-\alpha^{\prime}-\beta^{\prime}+\eta^{\prime}\right)}{\Gamma_{k}\left(\alpha^{\prime}-\sigma^{\prime}+\beta^{\prime}+k-\alpha^{\prime}-\beta^{\prime}\right) \Gamma_{k}\left(\alpha^{\prime}-\sigma^{\prime}+\beta^{\prime}+k+\eta^{\prime}\right)}, \\
\left(I_{y, \infty}^{\alpha^{\prime}, \beta^{\prime}, \eta^{\prime}} S^{\frac{\sigma^{\prime}}{k}-1}\right)_{k}(y)= & y^{\frac{\sigma^{\prime}-\beta^{\prime}}{k}-1} \frac{\Gamma_{k}\left(\beta^{\prime}-\sigma^{\prime}+k\right) \Gamma_{k}\left(-\sigma^{\prime}+k+\eta^{\prime}\right)}{\Gamma_{k}\left(-\sigma^{\prime}+k\right) \Gamma_{k}\left(\alpha^{\prime}-\sigma^{\prime}+\beta^{\prime}+k+\eta^{\prime}\right)} .
\end{aligned}
$$

\section{Generalized fractional integrals in terms of Wright functions}

In this section, we solve the composition of the Mittag-Leffler with power function to generalized left- and right-sided fractional integral operators and also discuss $k$-calculus.

Theorem 3 For $a, b, c, \rho, \delta \in \mathbb{C}$ with

$$
\Re(a)>0 \quad \text { and } \quad \Re(\rho+c-b)>0, \quad v>0, \quad \lambda>0, \quad w \in \mathbb{R}
$$

we have

$$
\begin{aligned}
\left(I_{0, u}^{a, b, c} t^{\rho-1} E_{v, \rho}^{\delta}\left(w t^{\lambda}\right)\right)(u)= & \frac{u^{-b-1+\rho}}{\Gamma(\delta)} \\
& \times{ }_{3} \Psi_{3}\left[\begin{array}{c}
(c-b+\rho, \lambda),(\rho, \lambda),(\delta, 1) \\
(a+c+\rho, \lambda),(\rho-b, \lambda),(\rho, v)
\end{array} \mid w u^{\lambda}\right] .
\end{aligned}
$$

Proof Using the power function and (10) in (6), we have

$$
\begin{aligned}
\left(I_{0, u}^{a, b, c} t^{\rho-1} E_{v, \rho}^{\delta}\left(w t^{\lambda}\right)\right)(u)= & \frac{u^{-a-b}}{\Gamma(a)} \int_{0}^{u}(u-t)^{a-1} \\
& \times{ }_{2} F_{1}\left(a+b,-c ; a ; 1-\frac{t}{u}\right) t^{\rho-1} \sum_{n=0}^{\infty} \frac{(\delta)_{n}}{\Gamma(v n+\rho) n !}\left(w t^{\lambda}\right)^{n} d t \\
= & \sum_{n=0}^{\infty} \frac{w^{n}(\delta)_{n}}{\Gamma(v n+\rho) n !}\left(I_{0, u}^{a, b, c} t^{\rho+\lambda n-1}\right)(u) .
\end{aligned}
$$


Since for $n=0,1,2, \ldots, \mathfrak{R}(\rho+\lambda n) \geq \mathfrak{R}(\rho+c-b)>0$, using Lemma 1 with $\rho$ replaced by $\rho+\lambda n$ in equation (38), we obtain

$$
\begin{aligned}
& \left(I_{0, u}^{a, b, c} t^{\rho-1} E_{v, \rho}^{\delta}\left(w t^{\lambda}\right)\right)(u) \\
& \quad=\frac{u^{\rho-b-1}}{\Gamma(\delta)} \sum_{n=0}^{\infty} \frac{\Gamma(\delta+n) \Gamma(\rho+\lambda n) \Gamma(c-b+\rho+\lambda n)}{\Gamma(-b+\rho+\lambda n) \Gamma(a+c+\rho+\lambda n) \Gamma(v n+\rho) n !}\left(w u^{\lambda}\right)^{n} .
\end{aligned}
$$

Using (16) in (39), we get

$$
\begin{aligned}
& \left(I_{0, u}^{a, b, c} t^{\rho-1} E_{v, \rho}^{\delta}\left(w t^{\lambda}\right)\right)(u)=\frac{u^{-b-1+\rho}}{\Gamma(\delta)} \\
& \times{ }_{3} \Psi_{3}\left[\begin{array}{c}
(c-b+\rho, \lambda),(\rho, \lambda),(\delta, 1) \\
(a+c+\rho, \lambda),(\rho-b, \lambda),(\rho, v)
\end{array} \mid w u^{\lambda}\right] .
\end{aligned}
$$

Theorem 4 For $a, b, c, \rho, \delta \in \mathbb{C}$ with

$$
\begin{aligned}
& \Re(a)>0 \quad \text { and } \quad \mathfrak{R}(a+\rho)>\max [-\Re(b),-\Re(c)], \quad \Re(b) \neq \Re(c), \\
& v>0, \quad \lambda>0, \quad w \in \mathbb{R},
\end{aligned}
$$

we have

$$
\begin{aligned}
\left(I_{u, \infty}^{a, b, c} t^{\rho-1} E_{\nu, \rho}^{\delta}\left(w t^{-\lambda}\right)\right)(u)= & \frac{u^{\rho-b-1}}{\Gamma(\delta)} \\
& \times{ }_{3} \Psi_{3}\left[\begin{array}{c}
(b-\rho+1, \lambda),(1+c-\rho, \lambda),(\delta, 1) \\
(1-\rho, \lambda),(a+b-\rho+c+1, \lambda),(\rho, \nu)
\end{array} \mid w u^{-\lambda}\right] .
\end{aligned}
$$

Proof Using the power function and (10) in (7), we have

$$
\begin{aligned}
\left(I_{u, \infty}^{a, b, c} t^{\rho-1} E_{v, \rho}^{\delta}\left(w t^{-\lambda}\right)\right)(u)= & \frac{1}{\Gamma(a)} \int_{u}^{\infty}(t-u)^{a-1} t^{-a-b} \\
& \times{ }_{2} F_{1}\left(a+b,-c ; a ; 1-\frac{u}{t}\right) t^{\rho-1} \sum_{n=0}^{\infty} \frac{(\delta)_{n}}{\Gamma(\nu n+\rho) n !}\left(w t^{-\lambda}\right)^{n} d t \\
= & \sum_{n=0}^{\infty} \frac{w^{n}(\delta)_{n}}{\Gamma(v n+\rho) n !}\left(I_{u, \infty}^{a, b, c} t^{\rho-\lambda n-1}\right)(u) .
\end{aligned}
$$

Since for $n=0,1,2, \ldots, \mathfrak{R}(\rho-\lambda n-1) \leq \Re(\rho+a-1)>1+\max [-\Re(b),-\Re(c)]$, using Lemma 2 with $\rho$ replaced by $\rho-\lambda n$, we reduce equation (40) to

$$
\begin{aligned}
& \left(I_{u, \infty}^{a, b, c} t^{\rho-1} E_{v, \rho}^{\delta}\left(w t^{-\lambda}\right)\right)(u) \\
& =\frac{u^{\rho-b-1}}{\Gamma(\delta)} \sum_{n=0}^{\infty} \frac{\Gamma(\delta+n) \Gamma(1-a+a+b+\lambda n-\rho) \Gamma(1-a-b+c+a+b+\lambda n-\rho)}{\Gamma(1-a-b+a+b+\lambda n-\rho) \Gamma(1+c+a+b+\lambda n-\rho) \Gamma(v n+\rho) n !} \\
& \quad \times\left(w u^{-\lambda}\right)^{n} .
\end{aligned}
$$


Using (16) in (41), we get

$$
\begin{aligned}
& \left(I_{u, \infty}^{a, b, c} t^{\rho-1} E_{v, \rho}^{\delta}\left(w t^{-\lambda}\right)\right)(u)=\frac{u^{\rho-b-1}}{\Gamma(\delta)} \\
& \times{ }_{3} \Psi_{3}\left[\begin{array}{c}
(b-\rho+1, \lambda),(1+c-\rho, \lambda),(\delta, 1) \\
(1-\rho, \lambda),(a+b-\rho+c+1, \lambda),(\rho, \nu)
\end{array} \mid w u^{-\lambda}\right] .
\end{aligned}
$$

Theorem 5 For $a, b, c, \rho, \delta \in \mathbb{C}$ with

$$
\Re(a)>0 \quad \text { and } \quad \Re(\rho+c-b)>0, \quad v>0, \quad \lambda>0, \quad w \in \mathbb{R},
$$

we have

$$
\begin{aligned}
\left(I_{0, u}^{a, b, c} t^{\frac{\rho}{k}-1} E_{k, v, \rho}^{\delta}\left(w t^{\frac{\lambda}{k}}\right)\right)(u)= & \frac{k^{1-\frac{\rho}{k}} u^{-b-1+\frac{\rho}{k}}}{\Gamma\left(\frac{\delta}{k}\right)} \\
& \times{ }_{3} \Psi_{3}\left[\begin{array}{c}
\left(c-b+\frac{\rho}{k}, \frac{\lambda}{k}\right),\left(\frac{\rho}{k}, \frac{\lambda}{k}\right),\left(\frac{\delta}{k}, 1\right) \\
\left(a+c+\frac{\rho}{k}, \frac{\lambda}{k}\right),\left(\frac{\rho}{k}-b, \frac{\lambda}{k}\right),\left(\frac{\rho}{k}, \frac{v}{k}\right)
\end{array} \mid k^{1-\frac{v}{k} w u^{\frac{\lambda}{k}}}\right] .
\end{aligned}
$$

Proof Using the power $k$-function and (11) in (6), we have

$$
\begin{aligned}
& \left(I_{0, u}^{a, b, c} t^{\frac{\rho}{k}-1} E_{k, v, \rho}^{\delta}\left(w t^{\frac{\lambda}{k}}\right)\right)(u) \\
& =\frac{u^{-a-b}}{\Gamma(a)} \int_{0}^{u}(u-t)^{a-1}{ }_{2} F_{1}\left(a+b,-c ; a ; 1-\frac{t}{u}\right) t^{\frac{\rho}{k}}-1 \sum_{n=0}^{\infty} \frac{(\delta)_{n, k}}{\Gamma_{k}(\nu n+\rho) n !}\left(w t^{\frac{\lambda}{k}}\right)^{n} d t \\
& =\sum_{n=0}^{\infty} \frac{w^{n}(\delta)_{n, k}}{\Gamma_{k}(v n+\rho) n !}\left(I_{0, u}^{a, b, c} t^{\frac{\rho+\lambda n}{k}-1}\right)_{k}(u) .
\end{aligned}
$$

Since for $n=0,1,2, \ldots, \mathfrak{R}(\rho+\lambda n) \geq \mathfrak{R}(\rho+c-b)>0$, using Lemma 1 with $\rho$ replaced by by $\frac{\rho+\lambda n}{k}$, we reduce equation (43) to

$$
\begin{aligned}
& \left(I_{0, u}^{a, b, c} t^{\frac{\rho}{k}-1} E_{k, v, \rho}^{\delta}\left(w t^{\lambda}\right)\right)(u) \\
& \quad=\frac{k^{1-\frac{\rho}{k}} u^{-b-1+\frac{\rho}{k}}}{\Gamma\left(\frac{\delta}{k}\right)} \sum_{n=0}^{\infty} \frac{\Gamma\left(\frac{\delta+n k}{k}\right) \Gamma\left(\frac{\rho+\lambda n}{k}\right) \Gamma\left(c-b+\frac{\rho+\lambda n}{k}\right)}{\Gamma\left(-b+\frac{\rho+\lambda n}{k}\right) \Gamma\left(a+c+\frac{\rho+\lambda n}{k}\right) \Gamma\left(\frac{v n+\rho}{k}\right) n !}\left(w k^{1-\frac{v}{k}} t^{\frac{\lambda}{k}}\right)^{n} .
\end{aligned}
$$

Using (16) in (44), we get

$$
\begin{aligned}
\left(I_{0, u}^{a, b, c} t^{\frac{\rho}{k}-1} E_{k, v, \rho}^{\delta}\left(w t^{\frac{\lambda}{k}}\right)\right)(u)= & \frac{k^{1-\frac{\rho}{k}} u^{-b-1+\frac{\rho}{k}}}{\Gamma\left(\frac{\delta}{k}\right)} \\
& \times{ }_{3} \Psi_{3}\left[\begin{array}{c}
\left(c-b+\frac{\rho}{k}, \frac{\lambda}{k}\right),\left(\frac{\rho}{k}, \frac{\lambda}{k}\right),\left(\frac{\delta}{k}, 1\right) \\
\left(a+c+\frac{\rho}{k}, \frac{\lambda}{k}\right),\left(\frac{\rho}{k}-b, \frac{\lambda}{k}\right),\left(\frac{\rho}{k}, \frac{v}{k}\right)
\end{array} \mid k^{1-\frac{v}{k}} w u^{\frac{\lambda}{k}}\right] .
\end{aligned}
$$

Remark 1 If we replace $k$ by one, then we get the result of [3].

Theorem 6 For $a, b, c, \rho, \delta \in \mathbb{C}$ with

$$
\mathfrak{N}(a)>0 \quad \text { and } \quad \mathfrak{R}(a+\rho)>\max [-\mathfrak{N}(b),-\mathfrak{R}(c)], \quad \mathfrak{R}(b) \neq \mathfrak{R}(c),
$$




$$
v>0, \quad \lambda>0, \quad w \in \mathbb{R},
$$

we have

$$
\begin{aligned}
& \left(I_{u, \infty}^{a, b, c} t^{\frac{\rho}{k}-1} E_{k, v, \rho}^{\delta}\left(w t^{\frac{-\lambda}{k}}\right)\right)(u) \\
& \quad=\frac{k^{1-\frac{\rho}{k}} u^{\frac{\rho-a-b}{k}+a-1}}{\Gamma\left(\frac{\delta}{k}\right)}{ }_{3} \Psi_{3}\left[\begin{array}{c}
\left(1+b-\frac{\rho}{k}, \frac{\lambda}{k}\right),\left(1+c-\frac{\rho}{k}, \frac{\lambda}{k}\right),\left(\frac{\delta}{k}, 1\right) \\
\left(1-\frac{\rho}{k}, \frac{\lambda}{k}\right),\left(1+a+b+c-\frac{\rho}{k}, \frac{\lambda}{k}\right),\left(\frac{\rho}{k}, \frac{v}{k}\right)
\end{array} \mid k^{1-\frac{v}{k}} w u^{\frac{-\lambda}{k}}\right] .
\end{aligned}
$$

Proof Using the power $k$-function and (11) in (7), we have

$$
\begin{aligned}
& \left(I_{u, \infty}^{a, b, c} t^{\frac{\rho}{k}-1} E_{k, v, \rho}^{\delta}\left(w t^{\frac{-\lambda}{k}}\right)\right)(u) \\
& =\frac{1}{\Gamma(a)} \int_{u}^{\infty}(t-u)^{a-1} t^{-a-b}{ }_{2} F_{1}\left(a+b,-c ; a ; 1-\frac{t}{u}\right) t^{\frac{\rho}{k}-1} \sum_{n=0}^{\infty} \frac{(\delta)_{n, k}}{\Gamma_{k}(v n+\rho) n !} \\
& \quad \times\left(w t^{\frac{-\lambda}{k}}\right)^{n} d t \\
& =\sum_{n=0}^{\infty} \frac{w^{n}(\delta)_{n, k}}{\Gamma_{k}(v n+\rho) n !}\left(I_{u, \infty}^{a, b, c} t^{\frac{\rho-\lambda n}{k}-1}\right)(u) .
\end{aligned}
$$

Since for $n=0,1,2, \ldots, \mathfrak{R}(\rho-\lambda n-1) \leq \mathfrak{R}(\rho+a-1)>1+\max [-\mathfrak{R}(b),-\Re(c)]$, using Lemma 2 with $\rho$ replaced by $\frac{\rho-\lambda n}{k}$, we reduce equation (47) to

$$
\begin{aligned}
& \left(I_{u, \infty}^{a, b, c} t^{\frac{\rho}{k}-1} E_{k, v, \rho}^{\delta}\left(w t^{\frac{-\lambda}{k}}\right)\right)(u) \\
& \quad=\frac{k^{1-\frac{\rho+v n}{k}} u^{\frac{\rho}{k}-b-1}}{\Gamma\left(\frac{\delta}{k}\right)} \sum_{n=0}^{\infty} \frac{\Gamma\left(\frac{\delta+n k}{k}\right) \Gamma\left(1+b-\frac{\rho-\lambda n}{k}\right) \Gamma\left(1+c-\frac{\rho-\lambda n}{k}\right)}{\Gamma\left(1-\frac{\rho-\lambda n}{k}\right) \Gamma\left(1+a+b+c-\frac{\rho-\lambda n}{k}\right) \Gamma\left(\frac{v n+\rho}{k}\right) n !}\left(k w u^{\frac{-\lambda}{k}}\right)^{n} .
\end{aligned}
$$

Using (16) in (48), we get

$$
\begin{aligned}
& \left(I_{u, \infty}^{a, b, c} t^{\rho-1} E_{v, \rho}^{\delta}\left(w t^{\frac{-\lambda}{k}}\right)\right)(u) \\
& \quad=\frac{k^{1-\frac{\rho}{k}} u^{\frac{\rho-a-b}{k}+a-1}}{\Gamma\left(\frac{\delta}{k}\right)}{ }_{3} \Psi_{3}\left[\begin{array}{c}
\left(1+b-\frac{\rho}{k}, \frac{\lambda}{k}\right),\left(1+c-\frac{\rho}{k}, \frac{\lambda}{k}\right),\left(\frac{\delta}{k}, 1\right) \\
\left(1-\frac{\rho}{k}, \frac{\lambda}{k}\right),\left(1+a+b+c-\frac{\rho}{k}, \frac{\lambda}{k}\right),\left(\frac{\rho}{k}, \frac{v}{k}\right)
\end{array} \mid k^{1-\frac{v}{k}} w u^{\frac{-\lambda}{k}}\right] .
\end{aligned}
$$

Remark 2 If we replace $k$ by one, then we get the result of [4].

\section{Euler transform for Mittag-Leffler function}

In this section, we investigate the Euler integral transformation for the Mittag-Leffler $k$ function. We also derive the Euler $k$-transformation of the Mittag-Leffler $k$-function.

Theorem 7 The Euler integral operator for the generalized Mittag-Leffler function is

$$
\left(I_{0}^{1} t^{a-1}(1-t)^{b-1} E_{v, \rho}^{\delta}\left(w t^{\lambda}\right)\right) d t=\frac{\Gamma(b)}{\Gamma(\delta)_{2}} \Psi_{2}\left[\begin{array}{c}
(\delta, 1),(a, \lambda) \\
(\rho, v),(a+b, \lambda)
\end{array} \mid w\right] .
$$


Proof

$$
\begin{aligned}
\left(I_{0}^{1} t^{a-1}(1-t)^{b-1} E_{v, \rho}^{\delta}\left(w t^{\lambda}\right)\right) d t & =\sum_{n=0}^{\infty} \frac{(\delta)_{n}}{\Gamma(v n+\rho) n !}(w)^{n} \int_{0}^{1} t^{a+\lambda n-1}(1-t)^{b-1} d t \\
& =\frac{\Gamma(b)}{\Gamma(\delta)} \sum_{n=0}^{\infty} \frac{\Gamma(\delta+n) \Gamma(a+\lambda n)}{\Gamma(a+b+\lambda n) \Gamma(v n+\rho) n !}(w)^{n} \\
& =\frac{\Gamma(b)}{\Gamma(\delta)_{2}} \Psi_{2}\left[\begin{array}{c}
(\delta, 1),(a, \lambda) \\
(\rho, v),(a+b, \lambda)
\end{array} \mid w\right] .
\end{aligned}
$$

Theorem 8 The Euler integral operator for the generalized Mittag Leffler $k$-function is

$$
\left(I_{0}^{1} t^{a-1}(1-t)^{b-1} E_{k, v, \rho}^{\delta}\left(w t^{\frac{\lambda}{k}}\right)\right) d t=\frac{\Gamma(b) k^{1-\frac{\rho}{k}}}{\Gamma(\delta)} \Psi_{2}\left[\begin{array}{c}
\left(\frac{\delta}{k}, 1\right),\left(a, \frac{\lambda}{k}\right) \\
\left(\frac{\rho}{k}, \frac{v}{k}\right),\left(a+b, \frac{\lambda}{k}\right)
\end{array} \mid k^{1-\frac{v}{k} w}\right] .
$$

Proof

$$
\begin{aligned}
\left(I_{0}^{1} t^{a-1}(1-t)^{b-1} E_{k, v, \rho}^{\delta}\left(w t^{\frac{\lambda}{k}}\right)\right) d t & =\sum_{n=0}^{\infty} \frac{(\delta)_{n, k}}{\Gamma_{k}(\nu n+\rho) n !}(w)^{n} \int_{0}^{1} t^{a+\frac{\lambda n}{k}-1}(1-t)^{b-1} d t \\
& =\frac{\Gamma(b) k^{1-\frac{\rho}{k}}}{\Gamma(\delta)} \sum_{n=0}^{\infty} \frac{\Gamma\left(\frac{\delta}{k}+n\right) \Gamma\left(a+\frac{\lambda n}{k}\right)}{\Gamma\left(a+b+\frac{\lambda n}{k}\right) \Gamma\left(\frac{v n}{k}+\frac{\rho}{k}\right) n !}\left(k^{1-\frac{v}{k}} w\right)^{n} \\
& =\frac{\Gamma(b) k^{1-\frac{\rho}{k}}}{\Gamma(\delta)} \Psi_{2}\left[\begin{array}{c}
\left(\frac{\delta}{k}, 1\right),\left(a, \frac{\lambda}{k}\right) \\
\left(\frac{\rho}{k}, \frac{v}{k}\right),\left(a+b, \frac{\lambda}{k}\right)
\end{array} \mid k^{1-\frac{v}{k} w}\right] .
\end{aligned}
$$

Theorem 9 Let $a, c, \rho, v, \lambda \in \mathbb{C}, w \in \mathbb{R}$, and $k \in \mathbb{R}^{+}$. Then the Euler $k$-transformation for the generalized Mittag Leffler $k$-function is

$$
\left(\frac{1}{k} I_{0}^{1} t^{\frac{a}{k}-1}(1-t)^{\frac{b}{k}-1} E_{k, v, \rho}^{\delta}\left(w t^{\frac{\lambda}{k}}\right)\right) d t=\frac{\Gamma_{k}(b)}{\Gamma_{k}(\delta)_{2}} \Psi_{2}^{k}\left[\begin{array}{c}
(\delta, k),(a, \lambda) \\
(a+b, \lambda),(\rho, v)
\end{array} \mid w\right] .
$$

Proof

$$
\begin{aligned}
\left(I_{0, k}^{1} t^{\frac{a}{k}-1}(1-t)^{\frac{b}{k}-1} E_{k, v, \rho}^{\delta}\left(w t^{\frac{\lambda}{k}}\right)\right) d t & =\sum_{n=0}^{\infty} \frac{(\delta)_{n, k}}{\Gamma_{k}(v n+\rho) n !} \frac{1}{k} \int_{0}^{1} t^{\frac{a}{k}-1}(1-t)^{\frac{b}{k}-1}\left(w t^{\frac{\lambda}{k}}\right)^{n} d t . \\
& =\sum_{n=0}^{\infty} \frac{(\delta)_{n, k} w^{n}}{\Gamma(v n+\rho) n !} \frac{1}{k} \int_{0}^{1}(t)^{\frac{a+\lambda n}{k}-1}(1-t)^{\frac{b}{k}-1} d t . \\
& =\frac{\Gamma_{k}(b)}{\Gamma_{k}(\delta)} \sum_{n=0}^{\infty} \frac{\Gamma_{k}(\delta+n k) \Gamma_{k}(a+\lambda n)}{\Gamma_{k}(a+b+\lambda n) \Gamma_{k}(v n+\rho) n !}(w)^{n} . \\
& =\frac{\Gamma_{k}(b)}{\Gamma_{k}(\delta)} \Psi_{2}^{k}\left[\begin{array}{c}
(a, \lambda),(\delta, k) \\
(a+b, \lambda),(\rho, v)
\end{array} \mid w\right] .
\end{aligned}
$$

\section{Conclusion}

In this paper, we have discussed two integral transforms involving the Gauss hypergeometric functions as their kernels. We have proved some composition formulae for these 
generalized fractional integrals with the Mittag-Leffler $k$-function. The results have been established in terms of the generalized Wright hypergeometric function. We have also developed the Euler integral $k$-transformation for the Mittag-Leffler $k$-function. Furthermore, if we take $k=1$, then we find out the classical results.

\begin{abstract}
Acknowledgements
The authors would like to thank the anonymous referee for his/her comments, which helped us improve this paper. The research work of Shahid Mubeen is supported by the Higher Education Commission of Pakistan under NRPU Project 2017.
\end{abstract}

Funding

Not applicable.

Availability of data and materials

Not applicable.

Competing interests

The authors declare that they have no competing interests.

Authors' contributions

Both authors SM and RSA contributed equally to write the manuscript. All authors read and approved the final manuscript.

\title{
Publisher's Note
}

Springer Nature remains neutral with regard to jurisdictional claims in published maps and institutional affiliations.

Received: 11 September 2019 Accepted: 9 December 2019 Published online: 16 December 2019

\section{References}

1. Khan, M.A., Ahmed, S.: On some properties of the generalized Mittag-Leffler function. SpringerPlus 2(1), 337 (2013)

2. Salim, T.O.: Some properties relating to the generalized Mittag-Leffler function. Adv. Appl. Math. Anal. 4(1), 21-30 (2009)

3. Salim, T.O., Faraj, A.W.: A generalization of Mittag-Leffler function and integral operator associated with fractional calculus. J. Fract. Calc. Appl. 3(5), 1-13 (2012)

4. Shukla, A.K., Prajapati, J.C.: On a generalization of Mittag-Leffler function and its properties. J. Math. Anal. Appl. 336(2), 797-811 (2007)

5. Srivastava, H.M., Tomovski, Z.: Fractional calculus with an integral operator containing a generalized Mittag-Leffler function in the kernel. Appl. Math. Comput. 211(1), 198-210 (2009)

6. Gehlot, K.S.: The generalized k-Mittag-Leffler function. Int. J. Contemp. Math. Sci. 7(45), 2213-2219 (2012)

7. Chand, M., Prajapati, J.C., Bonyah, E., Bansal, J.K.: Fractional calculus and applications of family of extended generalized Gauss hypergeometric functions. Discrete Contin. Dyn. Syst., Ser. S, 3053-3059 (2019)

8. Agarwal, P., Qi, F., Chand, M., Singh, G.: Some fractional differential equations involving generalized hypergeometric functions. J. Appl. Anal. 25(1), 37-44 (2019)

9. Chand, M., Agarwal, P., Hammouch, Z.: Certain sequences involving product of k-Bessel function. Int. J. Appl. Comput. Math. 4(4), 101 (2018)

10. Agarwal, P., Chand, M., Baleanu, D., O'Regan, D., Jain, S.: On the solutions of certain fractional kinetic equations involving k-Mittag-Leffler function. Adv. Differ. Equ. 2018(1), 249 (2018)

11. Chand, M., Hachimi, H., Rani, R.: New extension of beta function and its applications. Int. J. Math. Math. Sci. 2018, Article ID 6451592 (2018)

12. Agarwal, P., Chand, M., Choi, J., Singh, G.: Certain fractional integrals and image formulas of generalized k-Bessel function. Commun. Korean Math. Soc. 33(2), 423-436 (2018)

13. Chand, M., Rani, R.: Certain generating functions involving generalized Mittag-Leffler function. Int. J. Math. Anal. 12(6), 269-276 (2018)

14. Korpinar, Z., Inc, M., Baleanu, D., Bayram, M.: Theory and application for the time fractional Gardner equation with Mittag-Leffler kernel. J. Taibah Univ. Sci. 13(1), 813-819 (2019)

15. Tassaddiq, A., Khan, l., Nisar, K.S.: Heat transfer analysis in sodium alginate based nanofluid using MoS2 nanoparticles: Atangana-Baleanu fractional model. Chaos Solitons Fractals 130, 109445 (2020)

16. Khan, O., Khan, N., Baleanu, D., Nisar, K.S.: Computable solution of fractional kinetic equations using Mathieu-type series. Adv. Differ. Equ. 2019, 234 (2019)

17. Rahman, G., Abdeljawad, T., Khan, A., Nisar, K.S.: Some fractional proportional integral inequalities. J. Inequal. Appl. 2019(1), 244 (2019)

18. Shaikh, A.S., Nisar, K.S.: Transmission dynamics of fractional order typhoid fever model using Caputo-Fabrizio operator. Chaos Solitons Fractals 128, 355-365 (2019)

19. Huang, C.J., Rahman, G., Ghaffar, A., Qi, F.: Some inequalities via fractional conformable integral operators. J. Inequal. Appl. 2019, 217 (2019)

20. Shaikh, A., Tassaddiq, A., Nisar, K.S., Baleanu, D.: Analysis of differential equations involving Caputo-Fabrizio fractional operator and its applications to reaction diffusion equations. Adv. Differ. Equ. 2019, 178 (2019)

21. Bohner, M., Rahman, G., Mubeen, S., Nisar, K.: A further extension of the extended Riemann-Liouville fractional derivative operator. Turk. J. Math. 42(5), 2631-2642 (2018) 
22. Nisar, K.S., Suthar, D.L., Bohra, M., Purohit, S.D.: Generalized fractional integral operators pertaining to the by-product of Srivastava's polynomials and generalized Mathieu series. Mathematics 7(2), 206 (2019)

23. Araci, S., Rahman, G., Ghaffar, A., Nisar, K.S.: Fractional calculus of extended Mittag-Leffler function and its applications to statistical distribution. Mathematics 7(3), 248 (2019)

24. Rahman, G., Mubeen, S., Choi, J.: Certain extended special functions and fractional integral and derivative operators via an extended beta function. Nonlinear Funct. Anal. Appl. 24(1), 1-13 (2019)

25. Nisar, K.S., Mondal, S.R.: Pathway fractional integral operators involving k-Struve function. Afr. Math. 30, 1267-1274 (2019)

26. Lavault, C.: Fractional calculus and generalized Mittag-Leffler type functions (2017). arXiv:1703.01912v2 [math.CA]

27. Dumitru, B., Kai, D., Enrico, S.: Fractional Calculus: Models and Numerical Methods, vol. 3. World Scientific, Singapore (2012)

28. Nisar, K.S., Purohit, S.D., Mondal, S.R.: Generalized fractional kinetic equations involving generalized Struve function of the first kind. J. King Saud Univ., Sci. 28(2), 167-171 (2016)

29. Podlubny, I.: Fractional Differential Equations: An Introduction to Fractional Derivatives, Fractional Differential Equations, to Methods of Their Solution and Some of Their Applications, vol. 198. Elsevier, Amsterdam (1998)

30. Purohit, S.D.: Solutions of fractional partial differential equations of quantum mechanics. Adv. Appl. Math. Mech. 5(5), 639-651 (2013)

31. Purohit, S.D., Kalla, S.L.: On fractional partial differential equations related to quantum mechanics. J. Phys. A, Math. Theor. 44(4), $045202(2010)$

32. Uchaikin, V.V.: Fractional Derivatives for Physicists and Engineers, vol. 2. Springer, Berlin (2013)

33. Diaz, R., Pariguan, E.: On hypergeometric functions and Pochhammer k-symbol. Divulg. Mat. 15, 179-192 (2007)

34. Petojevic, A.: A note about the Pochhammer symbols. Math. Morav. 12, 37-42 (2008)

35. Kilbas, A.A., Sebastian, N.: Generalized fractional integration of Bessel function of the first kind. Integral Transforms Spec. Funct. 19, 869-883 (2008)

36. Saigo, M.: A remark on integral operators involving the Gauss hypergeometric functions. Kyushu Univ. 11, 135-143 (1978)

37. Samko, S.G., Kilbas, A.A., Marichev, O.I.: Fractional Integrals and Derivatives (1993). Translated from the 1987 Russian original

38. Srivastava, H.M., Choi, J.: Zeta and q-Zeta Functions and Associated Series and Integrals. Elsevier, Amsterdam (2012)

39. Rainville, E.D.: Special Functions, vol. 21. Macmillan Co., New York (1960)

40. Mittag-Leffler, G.M.: Sur la nouvelle fonction $E_{\alpha}(x)$. C. R. Acad. Sci. 137, 554-558 (1903)

41. Saxena, R.K., Mathai, A.M., Haubold, H.J.: On fractional kinetic equations. Astrophys. Space Sci. 282(1), $281-287$ (2002)

42. Wiman, A.: Über den Fundamentalsatz in der Teorie der Funktionen $E_{a}(X)$. Acta Math. 29(1), 191-201 (1905)

43. Prabhakar, T.R.: A singular integral equation with a generalized Mittag-Leffler function in the kernel. Yokohama Math. J. 19, 7-15 (1971)

44. Dorrego, G.A., Cerutti, R.A.: The k-Mittag-Leffler function. Int. J. Contemp. Math. Sci. 7(15), 705-716 (2012)

45. Gehlot, K.S., Prajapati, J.C.: Fractional calculus of generalized k-Wright function. J. Fract. Calc. Appl. 4(2), 283-289 (2013)

46. Rainville, E.D.: The Laplace Transform: An Introduction. Macmillan Co., New York (1963)

47. Ahmed, S: On the generalized fractional integrals of the generalized Mittag-Leffler function. SpringerPlus 3(1), 198 (2014)

48. Gupta, V., Bhatt, M.: Some results associated with k-hypergeometric functions. Int. J. Appl. Inf. Syst. 5(2), 106-109 (2015)

\section{Submit your manuscript to a SpringerOpen ${ }^{\circ}$ journal and benefit from:}

- Convenient online submission

- Rigorous peer review

- Open access: articles freely available online

- High visibility within the field

- Retaining the copyright to your article

Submit your next manuscript at $\boldsymbol{s p r i n g e r o p e n . c o m ~}$ 